\title{
Some ultrastructural observations of the vegetative, resting and excysting ciliate, urostyla grandis (urostylidae, hypotrichida)
}

\author{
ZHAO LIU1 ${ }^{1}$, LI YI-SONG ${ }^{1}$, LI JUN-GANG ${ }^{2}$, and GU FU-KANG ${ }^{1 *}$ \\ ${ }^{1}$ School of Life Science, East China Normal University, Shanghai 200062, China \\ ${ }^{2}$ Shanghai Institute of Applied Physics, Chinese Academy of Sciences, Shanghai 201800, China
}

\begin{abstract}
In order to reveal the differentiation characteristics of organelles of ciliates under different physiological status, the cellular ultrastructure of Urostyla grandis was studied by transmission electron microscopy. In the resting cells most ciliary shafts, kinetosomes and sub-pellicle microtubules were resorbed, and the endoplasmic reticulum (ER) disappeared with the autophagocytosis taking place within the cytoplasm. As well, the nuclear matrix of the macronucleus was extruded into the cytoplasm, forming pseudopodia-like structures with large quantities of heterochromatin $(\mathrm{CH})$ attached to the inner nuclear membrane. During excystment, membraneous structures developed and gradually increased in number to form the ER.

Autophagic vacuoles (AVs) appeared containing mitochondria, paraglycogen particles (PGP), membranous structures, etc. Moreover, the number of nucleoli decreased with the chromatin, condensing in parallel with the process of recombination. Based on these observations, it could be concluded that the de-differentiation of microtubular organelles and the changes occurring in macronuclei in the resting Urostyla grandis, as well as the differentiation of cytoplasmic organelles, digestion by AVs, and the recombination of chromatin during excystment, are not similar to events that occur in non-kinetosome-resorbing cysts (NKR).
\end{abstract}

Key terms: Urostyla grandis, Ultrastructure, Vegetative cell, Resting cell, Excysting cell.

Many hypotrichous ciliates can differentiate into three types of cysts when they face an adverse environment, and the excystment process can occur when the external environment is ameliorated ${ }^{[1-5]}$. In kinetosome-resorbing cysts, the cilia and sub-pellicle microtubules are fully resorbed. During excystment, the new structures regenerate spontaneously. Whereas, in nonkinetosome-resorbing cysts, the ciliary shafts of the cortical ciliatures are partially resorbed, and the new structures develop from the old ones during excystment ${ }^{[6-14]}$. Our results show that the structural redifferentiation characteristics of cellular organelles in kinetosome-resorbing cysts and non-kinetosome-resorbing cysts are not the same as those in urostyla type cysts. In urostyla type cysts, most of the ciliary shafts, kinetosomes and sub-pellicle microtubules are resorbed; and during excystment, the new structures develop from the partially preserved old ones.

In this study, the re-differentiation characteristics of cellular organelles, such as cilia, cyst wall, cytoplasmic organelles and nucleus during the excystment of Urostyla grandis were observed by transmission electron microscopy (TEM) to shed new light on the structural differentiation of ciliates.

\section{MATERIALS AND METHODS}

\subsection{Material}

Urostyla grandis was collected from a pond near Qingpu District, Shanghai, China, in October 2002. The collected ciliates were 
grown in the laboratory in culture dishes containing fermentation fluid of wheat grains at $20^{\circ} \mathrm{C}$. We then isolated a single cell from the dishes with a micropipette. After isolation, the cloned culture was established and maintained in the same conditions, with fermentation fluid as food source.

\subsection{Encystment and excystment}

At $20^{\circ} \mathrm{C}$, cysts formed completely 4 days after the concentration of Urostyla grandis attains a density of around 15000 cells $/ \mathrm{ml}$. When the old medium was replaced with a fresh one, the excystment process began within 1.5 to $2.0 \mathrm{~h}$, and lasted for about $12 \mathrm{~h}$.

\subsection{Collection of Urostyla grandis undergoing synchronous excystment}

The excystment process of Urostyla grandis was first observed by light microscope. The cysts appeared as opaque spheres, and then swelled slightly and their diaphaneity increased. With time, "the transparent belt" appeared beneath the cyst wall and broadened gradually. At the late phase of excystment, the ciliate started to rotate inside, and finally the redifferentiated ciliate were released through the broken cyst wall. These excystment characteristics were observed in synchronous collection of Urostyla grandis.

\subsection{Experimental design}

During the excystment process, three samples were taken from the excysting cells at 3,6 and $9 \mathrm{~h}$ for TEM observation. The vegetative cells and resting cysts were also observed by TEM as control groups. The sample preparations for TEM were published in our previous reports ${ }^{[10,15,16]}$.

\section{RESULTS}

\subsection{Ultrastructural characteristics of the vegetative cell}

\subsubsection{Pellicle and kinetosome}

Only a thin layer of pellicle could be observed around the vegetative cells. In some regions, it was difficult to distinguish the alveolar membranes from the inner and outer membranes (which stick together) at their inner rims. Beneath the pellicle, there was a tightly arranged layer of microtubules. The ciliary membrane was connected to the plasmalemma along a bristled area, where the kinetosome attached to the fundus of the pellicle (Fig. 1). Additionally, there was one or two cilium in dikinetid.

\subsubsection{Cytoplasmic organelles}

In vegetative cells, the endoplasmic reticulum structure was complex, with adhered ribosomes (Fig. 2). In addition, the oval food vacuole engulfed the residue of digestion, and their structure was simple (Fig. 3).

\subsubsection{Macronucleus}

Generally, the fusiform macronucleus was bounded by a typical double-layer envelope that was composed of 2 distinct membranes. Some of the chromatins were attached to the inner nuclear membrane, and $\mathrm{CHs}$ in the macronucleus showed an inhomogeneous distribution. The nucleoli, with larger size and lower electron density than the chromatin, were spherical in shape and embedded in the nuclear matrix. They were composed of granular and fibrous material (Fig. 4).

2.2 Ultrastructural characteristics of the resting and the excysting cell

\subsubsection{Cyst wall and cortex}

In the resting cyst, the wall was composed of three distinct layers. The outer layer (ectocyst) was thin and smooth, with low electron density. The granule layer (inner layer) varied in thickness and was composed of granular material, also with low electron density. The third layer (endocyst) was between them, displaying sawtooth-like folds, with high electron density (Fig. 5). Cilia and microtubules could not be observed in the cortex. 


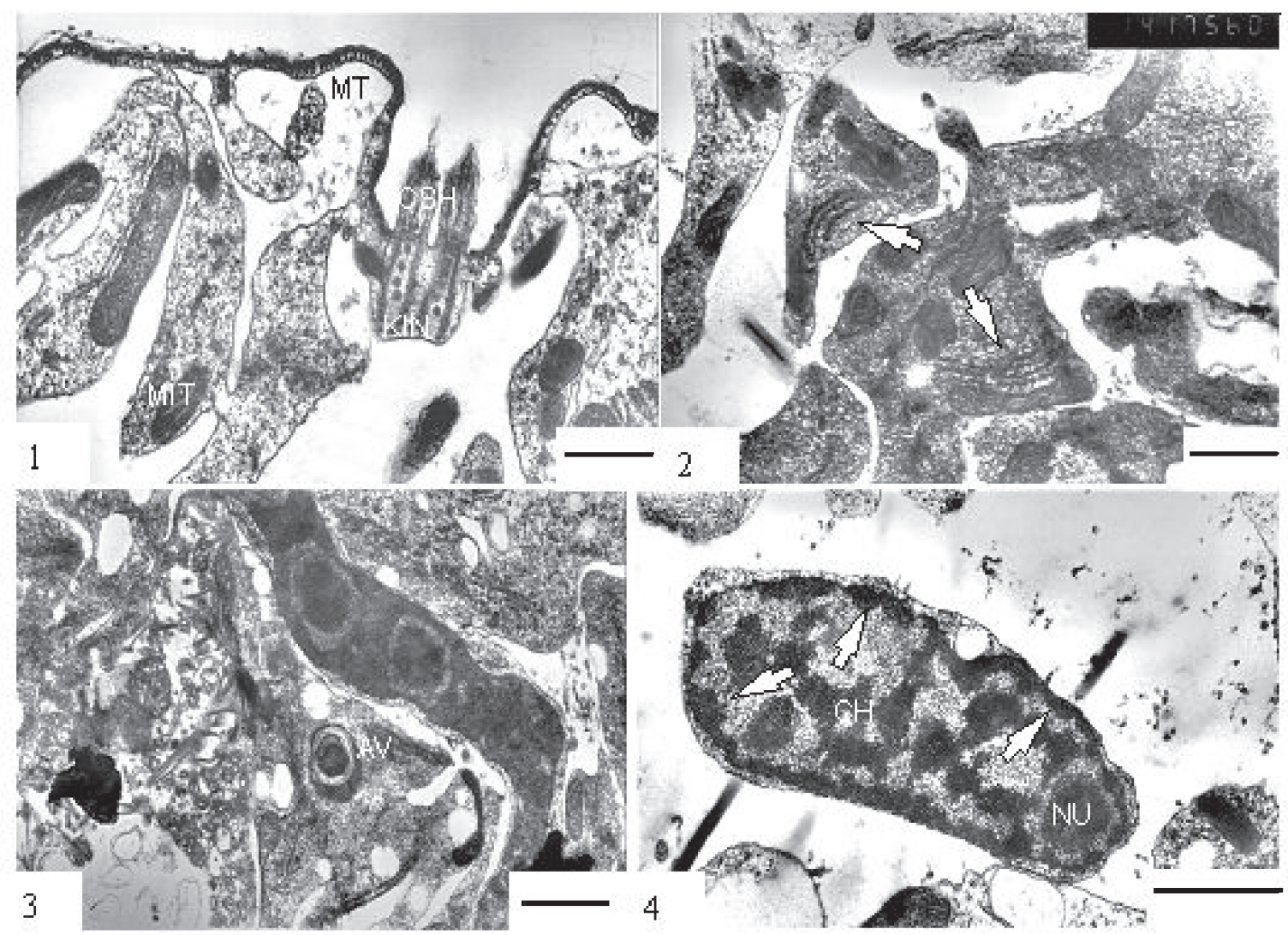

Fig. 1: Pellicle and sub-pellicular structures (CSH, ciliary shaft; KIN, kinetosome; MIT, mitochondrion; MT, microtubule layer); Fig. 2: Endoplasmic reticulum (ER), arrows show ER (The scale bar in Fig. 1 and Fig. 2 is $0.5 \mathrm{~mm}$ ); Fig. 3: Autophagic vacuole (AV); Fig. 4: Macronucleus (MAC), arrows show heterochromatin $(\mathrm{CH})$ attached to the inner membrane (The scale bar in Fig. 3 and Fig. 4 is $1 \mu \mathrm{m})$.

At 3-h of excystment, at several positions of the cyst wall, the space between the entocyte and the cyst wall became larger, and the remnant kinetosomes attached themselves to the inner cyst wall (Fig. 6). At 6-h, at certain positions of the endocyst wall, the granule layer started to break (Fig. 7). At 9-h, the endocyst wall became thinner and broke at several points. The cilia, microtubules and most of kinetosomes in the cortex appeared at this point (Figs. 8-9).

\subsubsection{Cytoplasmic organelles}

The membraneous structures could be observed at 6-h in the excysting cells (Fig. 10). At $9-\mathrm{h}$, these structures were further developed (Fig. 11).

In resting and excysting cells, autophagic vacuoles were easily observed.
They were circular or elliptic, located in the cytoplasm, and often bounded by doubledecked membrane. Because of different cellular organelles digested in them, their electron density was uneven. At 3-h, the paraglycogen particles and the remnant of mitochondria could be observed clearly in autophagic vacuoles (Fig. 12), while considerable vesicles were wrapped in autophagic vacuoles, which had an obvious difference in electron density, and connected mutually with the double-layer membrane (Fig. 13). At 6-h, lots of the paraglycogen particles were wrapped in autophagic vacuoles (Fig. 14). In addition, the vesicles in some autophagic vacuoles consisted of many concentric circles (Fig. 15). At 9-h, the internal structures of autophagic vacuoles were still complex (Fig. 16). 


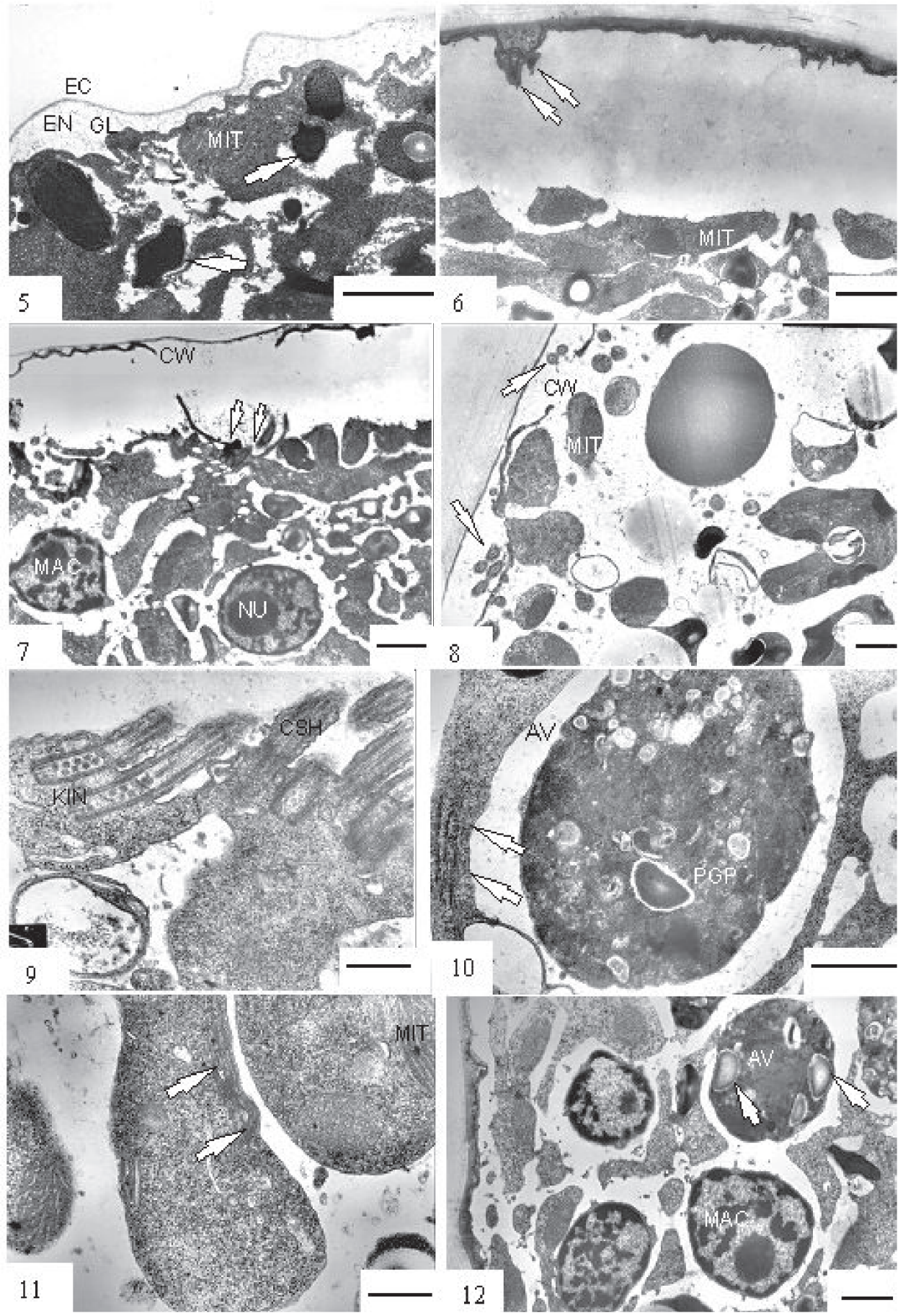

Fig. 5: cyst wall (EC, ectocyst; EN, endocyst; GL, granular layer.), residual kinetosomes and microtubules beneath the pellicle; Fig. 6: At 3-h, the spatium became larger, arrows show residual kinetosome; Fig. 7: At 6-h, partial positions of CW started to break, arrows show re-differentiation cilia; Fig. 8-9: At 9-h, several points of CW broke, most of cilia and kinetosomes appeared; Fig. 10: At 6-h, membraneous structures appeared, arrows show membraneous structures (The scale bar in Fig. 5-10is $1 \mu \mathrm{m}$ ); Fig. 11: At 9-h, membraneous structures were further developed, arrows show membraneous structures (scale bar $=0.5 \mu \mathrm{m}$ ); Fig. 12: At 3-h, remnant MIT and paraglycogen particles $(\mathrm{PGP})$ wrapped in AV, arrows show PGP in AV (scale bar $=1 \mathrm{~m} \mu)$. 


\subsubsection{Macronucleus}

In resting cysts, the macronucleus was circular (Fig. 17), while in excysting cells some of the macronuclei were dumbbellshaped (Figs. 18, 20) or spindle-shaped (Fig. 19).

In resting cysts, the nuclear membrane was extremely clear, with more heterochromatin attached to the inner membrane than in any other stage. No attached hetreochromatin nuclear pores could be clearly observed in sections of the nuclear membrane. Most of the chromatin within the nuclear is heterochromatin, condensed into lumps, two spherical nucleoli are fused together, and the fiber components in nucleoli were obviously lower in quantity than in other stages of development (Fig. 17). During excystment, the nuclear membrane became crumpled and indistinct. Sometimes, the chromatin condensed into belt-shaped structures and distributed between two spherical nucleoli (Fig. 18). A giant nucleolus could be observed in some macronuclei (Fig. 19). In addition, in excysting cells, the electron density of the nucleolus fiber component was far higher than in resting cysts.

\section{DISCUSSION}

\subsection{Ultrastructural changes of the ER}

At 6-h, some simple membraneous structures were observed in excysting cells. Subsequently, these structures were further developed. In vegetative cells, they developed into ER attached by the ribosomes. The results demonstrated that the ER is a transient cellular organelle, which is resorbed in cysts ${ }^{[17]}$ and goes through the process from simple to complex in excysting cells. Moreover, ER might relate to the synthesis of cellular materials, which are required during excystment process.

\subsection{Ultrastructural changes of $A V$}

In some species of hypotrichous ciliates, it has been reported that the AV in cysts can wrap different cellular organelles to digest them. For example, AV can wrap ciliary shafts in cysts of Euplotes encysticus ${ }^{[16]}$, membraneous structures in cysts of Oxytricha platystoma ${ }^{[15]}$, mitochondria in cysts of Paraurostyla weissei ${ }^{[18]}$ and axostyle-type structures in cysts of Pseudourostyla cristata ${ }^{[10]}$. The cellular organelles digested in AVs are considered as the main source of nutrients and energy of the vital activity in resting cysts ${ }^{[19]}$.

In the present study, in comparison to the simple structure of food vacuole in vegetative cells, the internal contents in $\mathrm{AVs}$ of resting and excysting cells were complex, such as different kinds of the cellular organelles and different degrees of digestion in them. At 3- and 6-h, the paraglycogen particles were observed wrapped in AVs, implying that this is a potential route through which the excysting Urostyla grandis gain energy by digesting the paraglycogen particles. At 6- and 9-h, there were considerable ribosomes located at the periphery of AVs. The energy of protein synthesis in these ribosomes was probably provided by digestion of cellular organelles, such as mitochondria, paraglycogen particles and membraneous structures in the AV. It seems that during Urostyla grandis excystment, they mainly utilize the nourishment and energy reserved in the AVs of their cysts. The features of AVs in urostyla type cysts are different from those of non-kinetosome-resorbing cysts. The AVs of the urostyla type cysts digested more kinds of organelles and materials.

\subsection{Ultrastructural changes of MAC}

The ultrastructural changes of MAC during encystment and excystment have been described in limited ciliate species. For example, two MAC fused during encystment in Stylonychia mytilus and Oxytrica fallax ${ }^{[5,20]}$, the quantity of DNA reduced first, then MAC underwent a duplication process in Euplotes encysticus ${ }^{[9]}$.

In Urostyla grandis, MAC was usually "shuttle"-shaped in vegetative cells, circular in resting cysts, while some of 


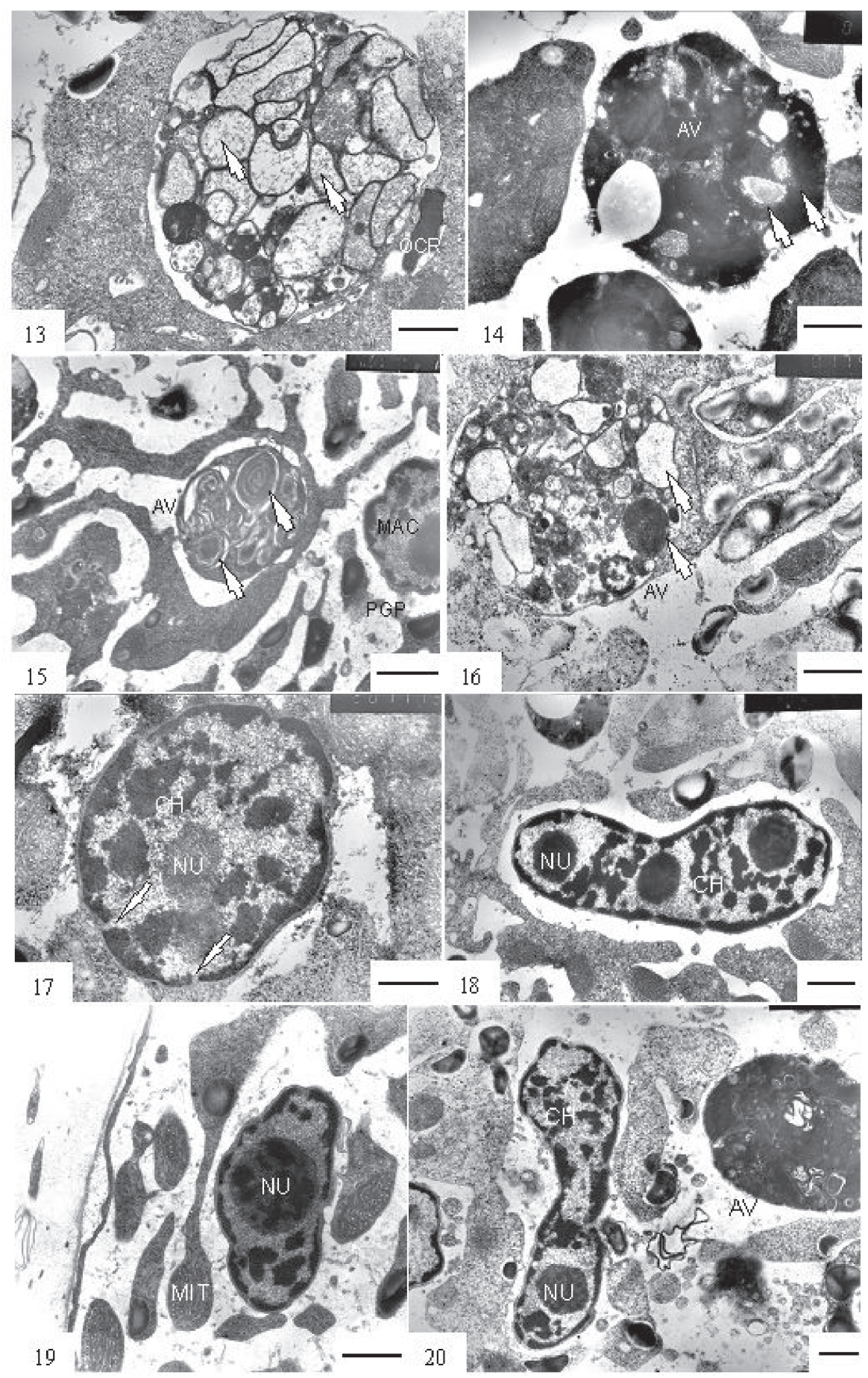

Fig. 13: At 3-h, lots of vesicles connected mutually with the double-layer membrane, arrows show vesicles in AV; Fig. 14: At 6-h, lots of PGP wrapped in AV, arrows show PGP in AV; Fig. 15: At 6-h, lots of concentric vesicles wrapped in AV, arrows show concentric vesicles in AV; Fig. 16: At 9-h, the complex internal structures of AV, arrows show the inclusion in AV (The scale bar in Fig. 12-16 is $1 \mu \mathrm{m}$ ); Fig. 17: MAC in resting cyst, arrows show nuclear pores (scale bar $=0.5 \mu \mathrm{m}$ ); Fig. 18: At 3-h, the belt-shaped chromatin distributed among spherical nucleoli (NU); Fig. 19: At 6-h, the giant nucleolus in MAC; Fig. 20: At 9-h, the dumbbell-shaped MAC (The scale bar in Fig. 1820 is $1 \mu \mathrm{m})$. 
them are dumbbell-shaped and spindleshaped during excystment. It suggests that MAC might adopt two patterns of division (binary fission and ternary fission) to recover their quantity. In resting cells, the fiber component of nucleoli had lower electron density than at points in time. During excystment, the middle parts of macronuclei were constricted and the number of nucleoli decreased, chromatin condensed into belt-shaped structures, and possible underwent the process of recombination.

In conclusion, in this paper we observed that the cyst wall became thinner and finally broke during the excysting process. The cilia, microtubules, most kinetosomes and AZM in the cortex, together with the tubular-type structure in cytoplasm, underwent a process from simple to complex. The AVs remained a complex structure during most excystment when compared to that in vegetative cells. Some of the macronuclei displayed a dumbbell or spindle shape during excystment. The redifferentiation characteristics of these cellular organs, reflected by their ultrastructural changes, are responsible for their possible functions associated with improvements in their external environment.

\section{ACKNOWLEDGMENTS}

This work was supported by the National Natural Science Foundation of China (No. 30772038).

\section{REFERENCES}

[1] CORLISS JD, ESSER SC (1974) Comments on the role of the cyst in the life cycle and survival of free-living protozoa. Trans Amer Micros Soc 93: 578-593.

[2] GUTIÉRREZ JC, MARTIN-GONZALES A, MATSUSAKA T (1990) Towards a generalized model of encystment (Cryptobiosis) in ciliates: a review and a hypothesis. Biosystrms 24: 17-24.

[3] GUTIÉRREZ JC, CALLEJAS S, BORNIQUEL S, BENITEZ L, MARTIN-GONZALES A (2001) Ciliate cryptobiosis: a microbial strategy against environmental starvation. Int Microbiol 4: 151- 157.
[4] CALVO P, FERNÁNDEZ-ALISEDA MC, GARRIDO J, TORRES A (2003) Ultrastructure, Encystment and Cyst Wall Composition of the Resting Cyst of the Peritrich Ciliate Opisthonecta henneguyi. J Eukaryot Microbiol 50(1): 49-56.

[5] WALKER GK, MAUGEL TK, GOODE D (1975) Some ultrastructural observations on encystment in Stylonychia mytilus (Ciliophora, Hypotrichida). Trans Amer Micros Soc 94: 147-154.

[6] WALKER GK, MAUGEL TK, GOODE D (1980a) Encystment and Excystment in hypotrich ciliates.! Gastrostyla steinii. Protistologica 4: 511-524.

[7] WALKER GK, MAUGEL TK, GOODE D (1980b) Encystment and Excystment in hypotrich ciliates.!a. Diophrys scutum remarks on comparative features. Protistologica 16: 525-531.

[8] RIOS RM, CALVO P, FEDRIANI C (1985) The cyst of Urostyla grandis (Hypotrichida: Urostylidae): ultrastructure and evolutionary implications. Protistologica 21: 481-485.

[9] GU FK, ZHANG ZR (1992) Studies on the macronucleus and micronucleus during encystment and excystment in Euplotes encysticus. Acta Zool Sin 38(2): 208-213.

[10] GU FK, NI B, YANG ZY, DU BJ (2002) Ultrastructure of the vegetative cell and resting cyst in Pseudourostyla cristata (Ciliophora, Hypotrichida). Acta Zool Sin 48(2): 251-257.

[11] GUTIÉRREZ JC, DÍAZ S, ORTEGA R, MARTINGONZALES A (2003) Ciliate resting cyst walls: a comparative review. Recent Res Dev Microbiol 7: 361379.

[12] ROSATI G, MODEO L, MELAI M, PETRONI G, VERNI F (2004) A multidisciplinary approach to describe protists: a morphological, ultrastructural, and molecular study on Peritromus kahli VilleneuveBrachon, 1940 (Ciliophora, Heterotrichea). J Eukaryot Microbiol 51: 49-59.

[13] FOISSNER W, MULLER H, WEISSE T (2005) The unusual, lepidosome-coated resting cyst of Meseres Corlissi (Ciliophora: Oligotrichea): light and scanning electron microsocopy, cytochemistry. Acta Protozool 44: 201-215.

[14] FOISSNER W (2005) The unusual, lepidosome-coated resting cyst of Meseres corlissi (Ciliophora: Oligotrichea): transmission electron microscopy and phylogeny. Acta Protozool 44: 217-230.

[15] GU FK, JI LM, NI B (1997) Observation on cellular ultrastructure during encystment of Oxytricha platystoma. Acta Zool Sin 43(3): 227-231.

[16] GU FK, NI B (1995) An ultrastructural study on resting cyst of Euplotes encysticus. Acta Biol Exer Sin 28(2): 163-171.

[17] GU FK (1991) Introduction of Protozoology. Higher Education Press, Beijing, pp: 52-57.

[18] GU FK, NI B, JI LM, SUI SG (1999) Some ultrastructural studies on resting cysts and their organelles in Paraurostyla weissei (Ciliophora, Hypotrichida). Zool Res 20(6): 406-410.

[19] CHEN L, NI B, GU FK (2000) Studies on electron microscopic enzymo-cytochemistry of organelles of resting cyst in Paraurostyla weissei (Ciliophora, Hypotrichida). Zool Res 21(3): 199-203.

[20] GRIMES W (1973) Differentiation during encystment and excystment in Oxytrica fallax. J Protozool 20: 92104. 
